Check for updates

Cite this: RSC Adv., 2017, 7, 46395

Received 22nd August 2017

Accepted 18th September 2017

DOI: 10.1039/c7ra09302k

rsc.li/rsc-advances

\section{Intracellular antioxidant effect of vanillin, 4-methylguaiacol and 4-ethylguaiacol: three components in Chinese Baijiu}

\author{
Dongrui Zhao, (D) ab Jinyuan Sun, ${ }^{* b c}$ Baoguo Sun, ${ }^{\text {bc }}$ Mouming Zhao, (D) ac \\ Fuping Zheng, ${ }^{\text {bc }}$ Mingquan Huang, ${ }^{\text {bc }}$ Xiaotao Sun ${ }^{\text {bc }}$ and Hehe Libc
}

\begin{abstract}
Vanillin, 4-methylguaiacol, and 4-ethylguaiacol, three bioactive components in Chinese Baijiu (Chinese liquor), were evaluated in terms of their possible antioxidant activities by DPPH, ABTS, ORAC, reducing power, metal chelation assays, and AAPH-induced HepG2 cell model. In in vitro chemical assays, vanillin, 4-methylguaiacol, and 4-ethylguaiacol exhibited stronger antioxidant activities than Trolox in ABTS, ORAC, and reducing power assays. Furthermore, three levels of treatment (1000, 500 and $10 \mathrm{mg} \mathrm{L}^{-1}$ ) of vanillin, 4-methylguaiacol, and 4-ethylguaiacol were assessed in the AAPH-induced HepG2 cell model. The results show that vanillin, 4-methylguaiacol, and 4-ethylguaiacol exerted similar antioxidant activities in a dose-dependent manner, and relieved the increased reactive oxygen species (ROS) quickly and efficiently; they also markedly improved AAPH-induced alterations in oxidative stress biomarkers and antioxidant enzymes, and were even more effective than Trolox. These findings indicate that vanillin, 4methylguaiacol, and 4-ethylguaiacol are potent natural antioxidants in Chinese Baijiu, and this lays the foundation for better illustrating the health benefits of Chinese Baijiu.
\end{abstract}

\section{Introduction}

Oxidative stress is currently a major health problem associated with the development of numerous diseases such as cancer, cardiovascular disorders, obesity, diabetes, ageing, and neurodegenerative diseases ${ }^{1}$ and is triggered by the excess formation of free radicals, which are generated by exogenous and endogenous processes., ${ }^{2,3}$ Under normal conditions, reactive oxygen species (ROS) play a beneficial role in the cellular response to infectious agents and the function of cellular signalling systems. However, excess ROS, triggered by a variety of factors such as immune cell activation, inflammation, mental or physical stress, excessive exercise, environmental pollution, smoking, certain drugs, and radiation, ${ }^{4-7}$ will lead to enhanced oxidative stress by an imbalance between the production of free radicals and endogenous antioxidant defenses and cause adverse effects to cells. ${ }^{\mathbf{8} 9}$ Finding effective ways to scavenge excess free radicals and keep free radicals at moderate levels has therefore become a subject of great interest.

${ }^{a}$ School of Food Science and Engineering, South China University of Technology, Guangzhou 510640, P. R. China

${ }^{b}$ Beijing Laboratory for Food Quality and Safety, Beijing Technology and Business University, Beijing 100048, P. R. China. E-mail: sunjinyuan@btbu.edu.cn; Fax: +8610-68984890; Tel: +86-10-68984890

'Beijing Advanced Innovation Center for Food Nutrition and Human Health, Beijing Technology and Business University, Beijing 100048, P. R. China
Phenolic compounds are natural antioxidants in foods and beverages, and have attracted considerable attention due to their possible beneficial effects on human health. There has been increasing evidence that phenolic compounds may protect cell constituents against oxidative damage and therefore prevent or delay various degenerative diseases associated with oxidative stress. ${ }^{10-12}$ Some studies have confirmed that phenolic compounds found in foods and beverages of plant origin (i.e., fruits, vegetables, grains, tea, coffee, and wine) relieve oxidative stress not only by acting as antioxidants themselves, but also by inducing intracellular antioxidative activity, such as via glutathione peroxidase (GSH-Px), catalase (CAT), and superoxide dismutase (SOD). ${ }^{\mathbf{1 0 , 1 3 - 1 5}}$ SOD, CAT, GSH-Px, and reduced glutathione (GSH) are the key participants of the antioxidant defense systems in cell. Specifically, SOD hastens the speed of dismutation of superoxide to hydrogen peroxide $\left(\mathrm{H}_{2} \mathrm{O}_{2}\right)$ and then comes the action of CAT, which transforms $\mathrm{H}_{2} \mathrm{O}_{2}$ into water and oxygen. For GSH, as one of the most important agents of the antioxidant defense system of the cell, it is well known that one of its functions is to prevent the harmful effects of oxidation by inhibiting the initiation of lipid peroxidation and scavenging free radicals and destroying them, which is catalyzed by GSHPx. ${ }^{16}$

Chinese Baijiu (Chinese liquor) is a traditional indigenous distilled spirit prepared from grain fermentation in China with 5000 years of history and is closely connected to the life of the Chinese people. To date, 1737 volatiles have been reported to be present in Chinese Baijiu, including alcohols, aldehydes, 
ketones, acids, esters, nitrogen-containing compounds, and sulfur-containing compounds. ${ }^{17}$ In ancient China, Baijiu was often considered as a drug rather than an alcoholic beverage. Previous studies have shown that moderate consumption of Baijiu is beneficial to human health; ${ }^{18}$ however, the relationship between Baijiu and health is very complicated due to its complex composition. Therefore, it is worthwhile to determine the bioactive components of Baijiu and their relationship with human health. Not only are phenolic compounds the important aromatic compounds found in Chinese Baijiu, ${ }^{\mathbf{1 7}}$ but they are also the components that present health benefits, and are therefore worthy of attention. In particular, vanillin (VA), 4methylguaiacol (4-MG), and 4-ethylguaiacol (4-EG), analogs of guaiacol, have been found in different types of Chinese Baijiu. ${ }^{17}$ Guaiacol reportedly possesses antioxidant activity due to its free radical scavenging activity.,19 It has been verified that ethyl vanillin (EVA), another analog of guaiacol, appears to decrease the enhanced ROS levels in the LPS-activated macrophage cells. ${ }^{12}$ Recent studies have shown that vanillin not only possesses antimicrobial activity ${ }^{20,21}$ and antioxidant activity, ${ }^{22}$ but exerts neuroprotective effects against harmaline-induced tremor. ${ }^{23}$ Therefore, 4-methylguaiacol and 4-ethylguaiacol may have potential antioxidant properties because of the similarity of their chemical structures (one oxidizable phenolic hydroxyl group with the presence of the methoxy group in ortho position to it, shown in Fig. 1) to guaiacol and vanillin. Although the aforementioned studies have confirmed the antioxidant activities of guaiacol, vanillin, and ethyl vanillin, ${ }^{3,22}$ there is still a lack of data supporting the antioxidant activities of 4-methylguaiacol and 4-ethylguaiacol. Moreover, because of different assays with different mechanisms, it is essential to evaluate the antioxidant activity of the components by multiple assay systems (i.e., in vitro chemical assays and cell models). However, no study has systematically evaluated the antioxidant activity of vanillin, 4methylguaiacol, and 4-ethylguaiacol using multiple assay systems.

In this study, the main objective was to evaluate the antioxidant activities of vanillin, 4-methylguaiacol, and 4-ethylguaiacol using DPPH, ABTS, ORAC, reducing power, and metal chelation assays. Their radical scavenging activities and protective effects against AAPH-induced oxidative damage in human liver cancer cells HepG2 were examined by determining the levels of oxidative stress biomarkers and antioxidant enzymes, including malondialdehyde (MDA), oxidized glutathione (GSSG), GSH, SOD, CAT, and GSH-Px.

\section{Materials and methods}

\section{Materials and reagents}

Herein, 6-hydroxy-2,5,7,8-tetramethylchroman-2-carboxylic acid (Trolox) and 1,1-diphenyl-2-picrylhydrazyl (DPPH) were obtained from Sigma-Aldrich (St. Louis, USA). Furthermore, 2,2'azino-bis (3-ethylbenzothiazoline-6-sulfonic acid) diammonium salt (ABTS) was purchased from Biotop Life Sciences Co. Ltd. (Beijing, China). Trichloroacetic acid (TCA) was purchased from Alfa Aesar (Lancashire, England) and 2,2'-azobis (2methylpropionamide)-dihydrochloride (AAPH) was obtained from Acros (Geel, Belgium). Potassium ferricyanide, iron(II) chloride tetrahydrate and potassium persulfate were obtained from Xilong Scientific Co. Ltd. (Beijing, China). Ferrozine, ethylenediaminetetraacetic acid disodium salt (EDTA $\mathrm{Na}_{2}$ ) and RIPA lysis buffer were purchased from Solarbio Life Sciences Co. Ltd. (Beijing, China). Fluorescein disodium (FL) was obtained from TCI (Shanghai) Development Co. Ltd. (Shanghai, China). Iron(III) chloride and absolute ethanol were obtained from Sinopharm Chemical Reagent Co. Ltd. (Beijing, China). All chemicals and reagents used were of analytical grade. Other chemicals and reagents are indicated in the specified methods.

Vanillin, 4-methylguaiacol, and 4-ethylguaiacol were purchased from Ruiyuan Spice Co. Ltd. (Zaozhuang, China). Microscale MDA, SOD, CAT, GSH-Px, and bicinchoninic acid (BCA) protein assay kits were purchased from Nanjing Jiancheng Institute of Biotechnology (Jiangsu, China). ROS and GSH/GSSG assay kits were purchased from Beyotime Institute of Biotechnology (Shanghai, China). Cell counting kit (CCK-8) assay was obtained from Dojindo (Kumamoto, Japan).

\section{DPPH assay}

DPPH radical scavenging activities were assessed as previously described, with some modifications. ${ }^{24}$ Briefly, $100 \mu \mathrm{L}$ of DPPH ethanolic solution $(0.15 \mathrm{mM})$ was mixed with $100 \mu \mathrm{L}$ working standard solution of sample (vanillin, 4-methylguaiacol, and 4ethylguaiacol) or $100 \mu \mathrm{L}$ Milli-Q water (Millipore, Bedford, MA,<smiles>COc1cc(C=O)ccc1O</smiles><smiles>COc1cc(C)ccc1O</smiles>

Vanillin
4-Methylguaiacol<smiles>CCc1ccc(O)c(OC)c1</smiles>

4-Ethylguaiacol<smiles>Cc1c(C)c2c(c(C)c1O)CCC(C)(C(=O)O)O2</smiles>

Trolox

Fig. 1 Chemical structures of vanillin, 4-methylguaiacol, 4-ethylguaiacol, and Trolox. 
USA) as the blank control. The mixture of $100 \mu \mathrm{L}$ ethanol and $100 \mu \mathrm{L}$ Milli-Q water was used in order to adjust the baseline $(A=0.000)$. The reaction was carried out at room temperature over $1 \mathrm{~h}$. The decrease in the DPPH radical concentration was monitored by measuring the absorbance at $517 \mathrm{~nm}$ with a spectrophotometer (Bio-Tek Instruments, Winooski, VT, USA). Trolox was used as the standard antioxidant, and the results were expressed as $\mu \mathrm{mol}$ Trolox equivalent (TE)/ $\mu \mathrm{mol}$ sample.

\section{ABTS assay}

The ABTS assay was conducted based on the previous method, with some modifications. ${ }^{25}$ Briefly, $\mathrm{ABTS}^{\cdot+}$ radical was prepared from a mixture of $7 \mathrm{mM}$ ABTS ammonium and $2.45 \mathrm{mM}$ potassium persulfate. The reaction was kept at room temperature for $12-16 \mathrm{~h}$ in the dark. Then, ABTS $^{\cdot+}$ radical was diluted with $100 \mathrm{mM}$ phosphate buffered saline (PBS) until it reached an absorbance near 0.70 at $734 \mathrm{~nm}$. Afterwards, $150 \mu \mathrm{L}$ of diluted $\mathrm{ABTS}^{\cdot+}$ radical was mixed with $50 \mu \mathrm{L}$ working standard solution of the sample (vanillin, 4-methylguaiacol, and 4-ethylguaiacol) or $50 \mu \mathrm{L}$ Milli-Q water as the blank control. The mixture of $150 \mu \mathrm{L} 100 \mathrm{mM}$ PBS and $50 \mu \mathrm{L}$ Milli-Q water was used in order to adjust the baseline $(A=0.000)$. After incubation for $30 \mathrm{~min}$ at room temperature, the absorbance at $734 \mathrm{~nm}$ was measured with a spectrophotometer. Trolox was used as the standard antioxidant, and the results were expressed as $\mu \mathrm{mol}$ Trolox equivalent (TE)/ $\mu$ mol sample.

\section{ORAC assay}

The modified ORAC assay was conducted according to the method described previously. ${ }^{26}$ Briefly, $120 \mu \mathrm{L}$ FL (120 nM) was mixed with $20 \mu \mathrm{L}$ working standard solution of the sample (vanillin, 4-methylguaiacol, and 4-ethylguaiacol) or $20 \mu \mathrm{L}$ MilliQ water as the blank control, and the mixture was pre-incubated at $37{ }^{\circ} \mathrm{C}$ for $15 \mathrm{~min}$. Then, $60 \mu \mathrm{L} \mathrm{AAPH}(40 \mathrm{mM})$ was added rapidly, using a multichannel pipet (Eppendorf, Hamburg, Germany). After being shaken for $30 \mathrm{~s}$, the fluorescence (excitation wavelength, $485 \mathrm{~nm}$; emission wavelength, $520 \mathrm{~nm}$ ) was monitored every $1 \mathrm{~min}$ for $100 \mathrm{~min}$ at $37^{\circ} \mathrm{C}$ by a spectrophotometer. Trolox was used as the standard antioxidant, and the results were expressed as $\mu \mathrm{mol}$ Trolox equivalent (TE) $/ \mu \mathrm{mol}$ sample.

\section{Reducing power}

Reducing power was determined according to a previous method..$^{25}$ Trolox was used as the standard antioxidant, and the results were expressed as $\mu \mathrm{mol}$ Trolox equivalent (TE)/ $\mu \mathrm{mol}$ sample.

\section{$\mathrm{Fe}^{2+}$ chelating ability assay}

The $\mathrm{Fe}^{2+}$ chelating ability was measured by a previously reported method. ${ }^{25}$ Ethylenediaminetetraacetic acid (EDTA) was used as the standard antioxidant, and the results were expressed as $\mu \mathrm{mol}$ EDTA equivalent (EE)/ $\mu \mathrm{mol}$ sample.

\section{Cell culture}

Human hepatic cell line HepG2 was provided by National Institute for Communicable Disease Control and Prevention, Chinese Center for Disease Control and Prevention (Beijing, China). Cells were grown and maintained in a humidified incubator at $37{ }^{\circ} \mathrm{C}$ and $5 \% \mathrm{CO}_{2}$ using Dulbecco's Modified Eagle's Medium (Mediatech, Manassas, VA, USA) supplemented with $10 \%(\mathrm{v} / \mathrm{v})$ fetal bovine serum, which was obtained from Gibco (Carlsbad, CA, USA). All studies were performed using $80 \%$ confluent cells before treatment.

\section{Measurement of cell viability}

HepG2 cells $\left(1 \times 10^{5}\right)$ were grown on a 96-well microplate. Cell viability, after treatment with various concentrations of sample (vanillin, 4-methylguaiacol, and 4-ethylguaiacol), was assessed by CCK-8 assay. ${ }^{27}$ After designated treatments, $10 \mu \mathrm{L}$ of CCK-8 solution was added to the cells cultured in 96-well plates, and OD values were measured at $450 \mathrm{~nm}$ using a spectrophotometer. Cell viability was expressed as a percentage of the absorbance of untreated control cells.

\section{AAPH-induced ROS inhibition assay}

HepG2 cells $\left(1 \times 10^{5}\right)$ were grown on a 96-well microplate. After incubation for $24 \mathrm{~h}$ at $37{ }^{\circ} \mathrm{C}\left(5 \% \mathrm{CO}_{2}\right)$, the growth medium was removed and the wells were washed with serum-free medium. The cells were then treated for $1 \mathrm{~h}$ with $100 \mu \mathrm{L}$ of serum-free medium containing $10 \mu \mathrm{mol} \mathrm{L}{ }^{-1}$ DCFH-DA with the sample (vanillin, 4-methylguaiacol, 4-ethylguaiacol, and Trolox) and without the sample. Afterwards, the medium was removed and the wells were washed with serum-free medium. Then, $100 \mu \mathrm{L}$ serum-free medium containing $200 \mu \mathrm{M}$ AAPH was added, and the mixture was incubated at $37{ }^{\circ} \mathrm{C}\left(5 \% \mathrm{CO}_{2}\right)$ for up to $3 \mathrm{~h}$. The cells treated with AAPH alone were denoted as the AAPH group. The cells treated with DCFH-DA only were mixed with $100 \mu \mathrm{L}$ of serum-free medium as the blank control. The fluorescence (excitation wavelength, $488 \mathrm{~nm}$; emission wavelength, $525 \mathrm{~nm}$ ) of the mixture was measured using a spectrophotometer. Batches of the reaction mixture were monitored after incubation of $0,30,60,120,180 \mathrm{~min}$ at $37^{\circ} \mathrm{C}$.

\section{Measurement of MDA, GSH, GSSG and BCA content}

HepG2 cells $\left(1 \times 10^{5}\right)$ were grown on a 24 -well microplate. After incubation of $24 \mathrm{~h}$ at $37{ }^{\circ} \mathrm{C}\left(5 \% \mathrm{CO}_{2}\right)$, the growth medium was removed and the serum-free medium containing different amounts $\left(1000,500\right.$ and $10 \mathrm{mg} \mathrm{L}^{-1}$ ) of sample (vanillin, 4-methylguaiacol, and 4-ethylguaiacol) or Trolox (500 $\left.\mathrm{mg} \mathrm{L}^{-1}\right)$ was added to the well. After being treated for $2 \mathrm{~h}\left(37^{\circ} \mathrm{C}, 5 \%\right.$ $\mathrm{CO}_{2}$ ), the medium was removed, and the wells were washed with serum-free medium. Then, serum-free medium containing $200 \mu \mathrm{M} \mathrm{AAPH}$ was added and the mixture was incubated at $37{ }^{\circ} \mathrm{C}\left(5 \% \mathrm{CO}_{2}\right)$ for up to $2 \mathrm{~h}$. The cells treated with AAPH alone were denoted as the AAPH group, and untreated cells were the blank control group. Afterwards, the medium was removed, and the wells were washed three times with PBS (Gibco, Carlsbad, CA, USA). The cells were then digested by trypsin (Gibco, 
Carlsbad, CA, USA) and lysed with RIPA lysis buffer and centrifuged at $14000 \mathrm{~g}$ for $10 \mathrm{~min}$ at $4{ }^{\circ} \mathrm{C}$. Afterwards, the cell supernatant was collected and used to measure the content of MDA, GSH, GSSG and BCA, according to the assay kit. The results are presented as relative to the control group value.

\section{Measurement of CAT, SOD and GSH-Px activity}

The cell supernatant obtained by the same method mentioned in the previous section was used to determine the activity of CAT, SOD and GSH-Px, according to the assay kit. The results were expressed as values relative to the control group.

\section{Statistical analysis}

Two independent experiments were performed at least in triplicate. The data were expressed as mean \pm standard deviation (SD). Statistical differences between groups were obtained using the one-way ANOVA test with a post hoc Tukey test. $p<0.05$ was accepted as statistically significant.

\section{Results}

\section{Antioxidant activity measured by DPPH, ABTS, ORAC, reducing power and $\mathrm{Fe}^{2+}$ chelating ability assays}

DPPH, ABTS, ORAC, reducing power, and $\mathrm{Fe}^{2+}$ chelating ability assays were applied to evaluate the antioxidant activities of vanillin (VA), 4-methylguaiacol (4-MG), and 4-ethylguaiacol (4-EG). Trolox was used as the standard. The chemical structures of these compounds are shown in Fig. 1. Previously, Prior et $a{ }^{28}{ }^{28}$ reported that DPPH was a stable nitrogen radical, and many antioxidants that reacted quickly with other free radicals may react slowly or may even be inert to DPPH. Therefore, the effect of reaction time on the DPPH radical scavenging rate of vanillin, 4-methylguaiacol, and 4-ethylguaiacol was measured. As shown in Fig. 2, VA clearly, exhibited the weakest activity (scavenging rate at $60 \mathrm{~min}, 18.25 \%$ ) in the DPPH radical assay, and no significant difference was found in the scavenging rate among the different reaction times, except for that at $0 \mathrm{~min}$.

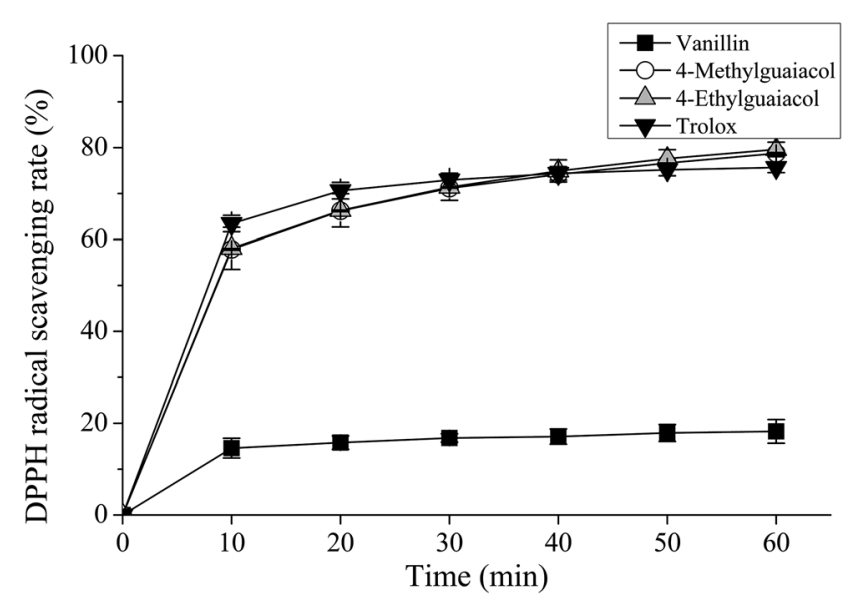

Fig. 2 Time courses of DPPH radical scavenging activities of vanillin, 4-methylguaiacol, 4-ethylguaiacol, and Trolox. Data are shown as the mean \pm SD from three independent experiments.
Although the DPPH radical scavenging rates of 4-MG $(78.76 \%)$ and 4-EG $(79.62 \%)$ were slightly higher than that of Trolox $(75.67 \%)$ at $60 \mathrm{~min}, 4-\mathrm{MG}, 4-\mathrm{EG}$, and Trolox showed nearly the same reaction profiles, reacting rapidly between 0 and $10 \mathrm{~min}$, and then slowing down. Finally, $40 \mathrm{~min}$ was defined as the reaction time in the DPPH assay due to no significant difference being detected after $40 \mathrm{~min}$.

Fig. 3 shows the antioxidant activities of VA, 4-MG, and 4-EG determined in DPPH, ABTS, ORAC, and reducing power assays. In the DPPH assay, VA displayed very little DPPH radical scavenging activity, with TE value of $0.23 \mu \mathrm{mol} \mathrm{TE} \mu \mathrm{mol}^{-1} \mathrm{VA}$, which is consistent with a previous study. ${ }^{22}$ However, 4-MG and 4-EG showed stronger DPPH radical scavenging activities with TE

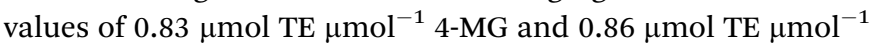
4-EG, almost four times that of VA's TE value. In the ABTS assay, VA, 4-MG and 4-EG exhibited stronger radical scavenging activity than Trolox. Furthermore, 4-EG had the highest activity with a TE value of $4.30 \mu \mathrm{mol}$ TE $\mu \mathrm{mol}^{-1} 4$-EG, followed by 4-MG and VA with TE values of 3.63 and $1.36 \mu \mathrm{mol}$ TE $\mu \mathrm{mol}^{-1}$, respectively. In the ORAC assay, interestingly, VA showed the strongest radical scavenging activity with a TE value of 1.59 $\mu \mathrm{mol}$ TE $\mu \mathrm{mol}^{-1} \mathrm{VA}$, followed by 4-EG and 4-MG with TE values of 1.39 and $0.94 \mu \mathrm{mol} \mathrm{TE} \mu \mathrm{mol}^{-1}$, respectively. In the reducing power assay, VA, 4-MG, and 4-EG exhibited stronger antioxidant activity than Trolox. VA is the strongest antioxidant with a TE value of $1.70 \mu \mathrm{mol} \mathrm{TE} \mu \mathrm{mol}^{-1} \mathrm{VA}$; 4 -EG had the lowest TE value of $1.20 \mu \mathrm{mol} \mathrm{TE} \mu \mathrm{mol}^{-1}$ 4-EG, while 4 -MG had a moderate TE value of $1.58 \mu \mathrm{mol} \mathrm{TE} \mu \mathrm{mol}^{-1} 4$-MG. As for their $\mathrm{Fe}^{2+}$ chelating abilities, none of them showed $\mathrm{Fe}^{2+}$ chelating ability with $\mathrm{EE}$ values less than $0.01 \mu \mathrm{mol} \mathrm{EE} \mu \mathrm{mol}^{-1}$.

\section{Cell viability}

In order to determine the treatment of samples, the cell viability of HepG2 cells was measured with CCK-8 assay. As shown in Fig. 4, although there remained discrepancies in samples of different treatment contents, regularity was obtained. High

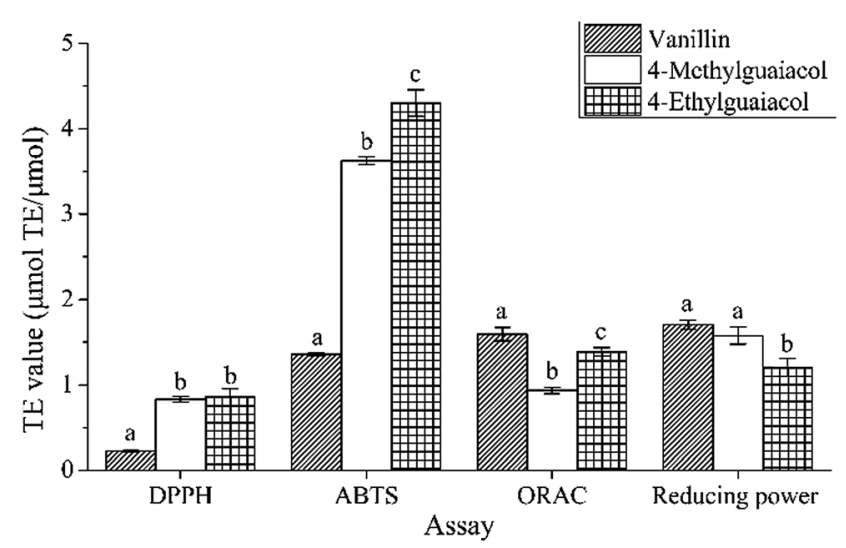

Fig. 3 Antioxidant activities of vanillin, 4-methylguaiacol, and 4-ethylguaiacol determined in DPPH, ABTS, ORAC, and reducing power assays. Data are shown as the mean \pm SD from three independent experiments. Different letters represent the significant difference at $p<$ 0.05 . 

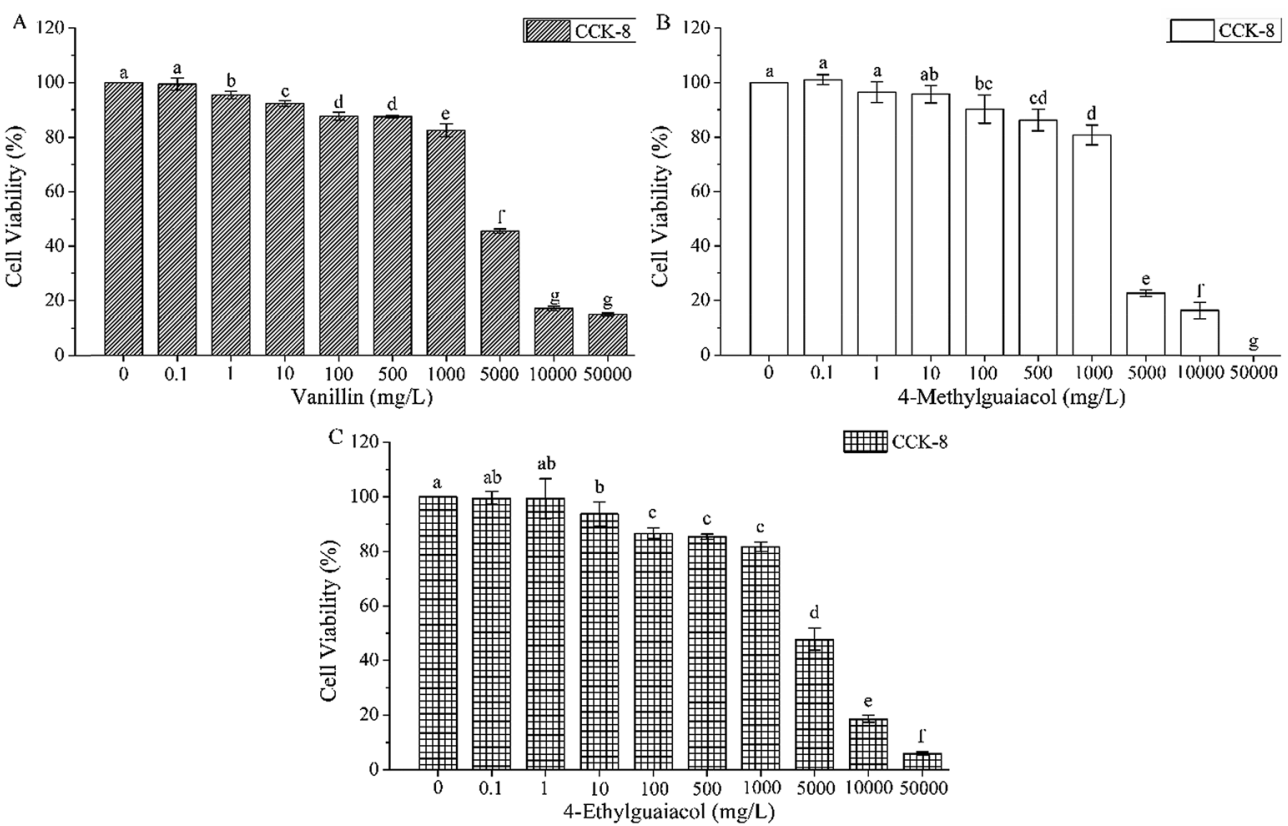

Fig. 4 Cell viability of HepG2 cells treated with various concentrations of vanillin (A), 4-methylguaiacol (B), and 4-ethylguaiacol (C). Data are shown as the mean \pm SD from three independent experiments. Different letters represent the significant difference at $p<0.05$.

concentrations of sample treatment had significant cytotoxic effects on HepG2 cells, resulting in decreased CCK-8 activity. The cell viability was above $80 \%$ when the treatment contents of samples were below $5000 \mathrm{mg} \mathrm{L}^{-1}$; therefore, three levels $(1000$, 500 , and $10 \mathrm{mg} \mathrm{L}^{-1}$ ) of inoculation content were applied in the AAPH-induced ROS inhibition assay.

\section{Protective effects of vanillin, 4-methylguaiacol and 4-} ethylguaiacol on AAPH-induced ROS in HepG2 cells

Peroxyl radicals generated from the decomposition of AAPH at $37{ }^{\circ} \mathrm{C}$ could attack the cell membrane to induce lipid and protein peroxidation and thus generate ROS and accelerate cells apoptosis. ${ }^{29}$ ROS are believed to play an important role in a variety of physiological processes; hence, the protective effects of vanillin (VA), 4-methylguaiacol (4-MG), and 4-ethylguaiacol (4-EG) against AAPH-induced ROS in HepG2 cells were determined, and the treatment content and incubation times of samples were taken into consideration. Trolox was used as the standard. As shown in Fig. 5, the amount of ROS increased rapidly between 0 and $30 \mathrm{~min}(p<0.05)$, except for the control group, which was very stable with limited ROS observed for up to $180 \min (155.79 \pm 1.58, p>0.05)$. Moreover, it was obvious that ROS was generated in a time-dependent manner. After $30 \mathrm{~min}$, the ROS amount of the AAPH-induced group (AAPH group) grew at a steady rate until $180 \mathrm{~min}(301.15 \pm 3.02)$. However, the AAPH-induced sample incubation groups (sample groups) all decreased significantly in their ROS amounts (in the range from $151.88 \pm 1.60$ to $242.85 \pm 2.32$ at $60 \mathrm{~min}$ ) within 30 to $60 \mathrm{~min}(p<0.05)$. Afterwards, most of the sample groups showed various degrees of increased ROS (in the range from $153.05 \pm 3.08$ to $268.33 \pm 2.00$ at $180 \mathrm{~min}$ ). As for the ROS amounts of Trolox and sample groups at the same level
(500 $\mathrm{mg} \mathrm{\textrm {L } ^ { - 1 }}$ ), no significant difference was obtained between Trolox and sample groups before $60 \mathrm{~min}$, except for the 4-EG group at $30 \mathrm{~min}$, the ROS amount of which $(216.67 \pm 3.48)$ was remarkably lower than that $(236.30 \pm 4.36)$ of the Trolox group $(p<0.05)$. However, the ROS amount of Trolox was significantly higher than those of sample groups after $60 \mathrm{~min}(p<0.05)$.

Also noted was that VA, 4-MG, and 4-EG exhibited dosedependence in protecting HepG2 cells from AAPH-induced ROS. As shown in Fig. 5, when the HepG2 cells were treated with AAPH alone, the intracellular ROS level increased by about 2 -fold $(p<0.05$ ), even though low level of sample treatment content (10 $\mathrm{mg} \mathrm{L}^{-1}$ ) was able to remarkably suppress the ROS generation elevated by AAPH in the HepG2 cells $(p<0.05)$. Furthermore, with the increase of treatment content, the suppressing ability of the sample increased significantly $(p<0.05)$, particularly that of the high level treatment group of the 4-EG (1000 $\mathrm{mg} \mathrm{L}^{-1}$ treated group), which suppressed the ROS level to just lower than the control group after $180 \mathrm{~min}$. Taken together, 4-ethylguaiacol had the highest protective effect with the lowest ROS amount $(153.05 \pm 3.08)$ in the high level treatment group (1000 $\mathrm{mg} \mathrm{L}^{-1}$ ), followed by 4-methylguaiacol (169.92 \pm 1.19$)$ and vanillin (186.77 \pm 1.20$)$.

\section{Effects of vanillin, 4-methylguaiacol and 4-ethylguaiacol on the AAPH-induced changes in MDA, GSSG, GSH, CAT, SOD, and GSH-Px levels of HepG2 cells}

In order to gain deep insight into the antioxidant activities of vanillin (VA), 4-methylguaiacol (4-MG), and 4-ethylguaiacol (4EG), the important oxidative stress markers and antioxidant enzymes in HepG2 cells were taken into consideration. MDA is an end product of membrane lipid peroxidation, which can interact with DNA and proteins. ${ }^{30}$ As a highly toxic molecule, its 

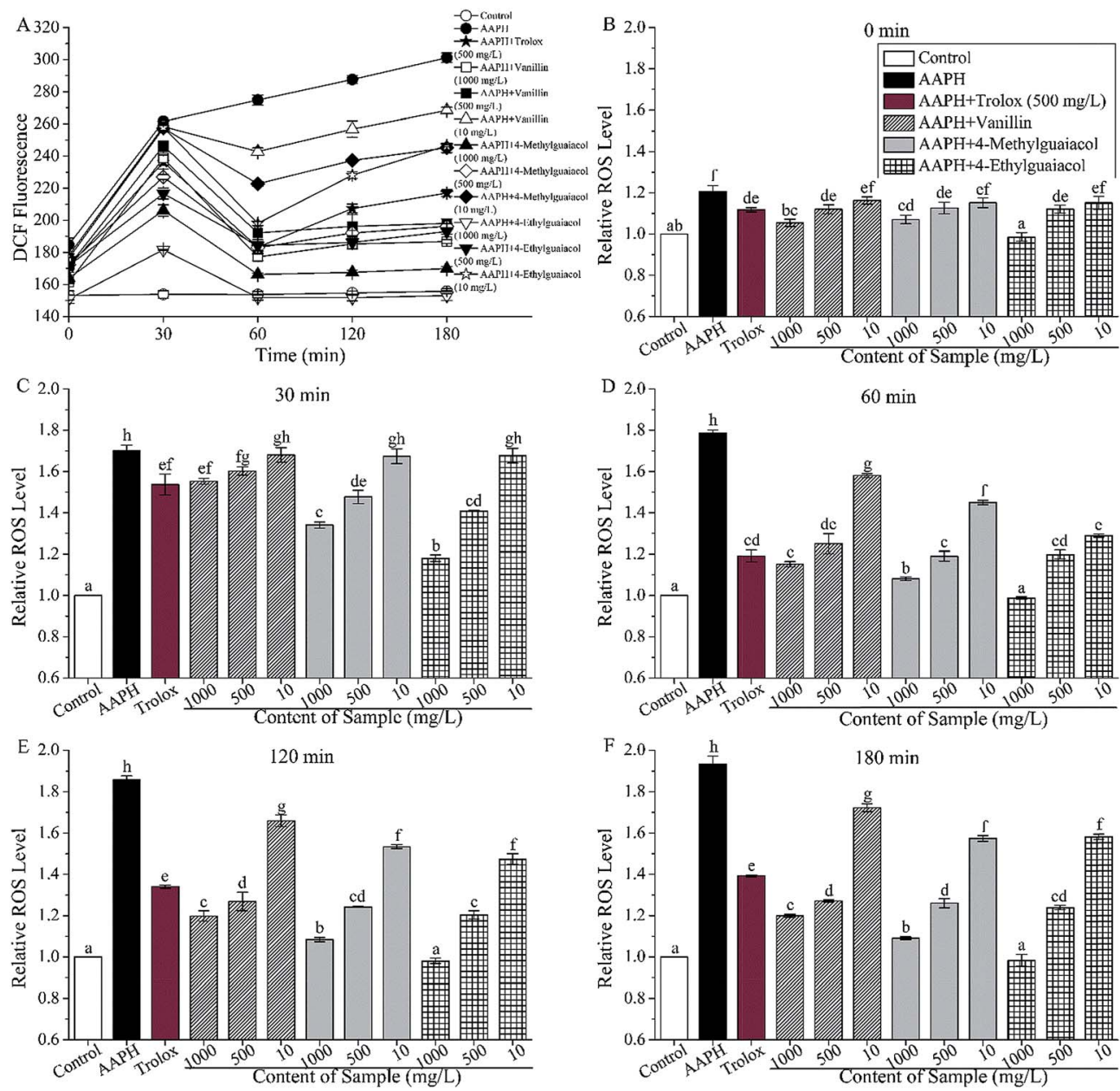

Fig. 5 Protective effects of vanillin, 4-methylguaiacol, and 4-ethylguaiacol against AAPH-induced ROS in HepG2 cells. The ROS level of all groups within $180 \mathrm{~min}$ are shown in (A). The results of different incubation time (0 min (B), $30 \mathrm{~min}(C), 60 \mathrm{~min}(\mathrm{D}), 120 \mathrm{~min}(\mathrm{E})$, and $180 \mathrm{~min}(\mathrm{~F}))$ are expressed as relative values versus the control group. Data are shown as the mean \pm SD from three independent experiments. Different letters represent the significant difference at $p<0.05$.

interaction with DNA and proteins has often been referred to as potentially mutagenic and atherogenic. ${ }^{31} \mathrm{MDA}$ is therefore widely used as a biomarker for oxidative stress. As shown in Fig. 6A, treatment of AAPH significantly caused the formation of MDA in HepG2 cells $(p<0.05)$. The MDA content of the AAPH group increased 2.83 times, compared to the control group. However, the MDA contents of sample groups were significantly decreased by $34.70-75.21 \%$, compared to that of the AAPH group $(p<0.05)$. At high level treatment $\left(1000 \mathrm{mg} \mathrm{L}^{-1}\right)$, no significant difference in the MDA content was found among VA, 4-MG, 4-EG and control groups. Moreover, there was no significant difference observed for HepG2 cells treated with medium levels $\left(500 \mathrm{mg} \mathrm{L}^{-1}\right.$ ), although their suppressing abilities on the formation of MDA were weaker than high level treatment $\left(1000 \mathrm{mg} \mathrm{L}^{-1}\right)$. However, at low level treatment $\left(10 \mathrm{mg} \mathrm{L}^{-1}\right)$, VA showed the highest inhibition capability (content of MDA, $\left.0.98 \pm 0.02 \mathrm{nmol} \mathrm{mg}_{\text {prot }^{-1}}\right)$ with remarkable difference $(p<0.05)$, compared to 4-EG $\left(1.31 \pm 0.03 \mathrm{nmol} \mathrm{mg}_{\text {prot }}{ }^{-1}\right)$ and 4-MG $\left(1.64 \pm 0.04 \mathrm{nmol} \mathrm{mg}_{\text {prot }}{ }^{-1}\right)$. In addition, VA, 4-MG, and 4-EG showed stronger inhibition capabilities toward MDA than Trolox (content of MDA, $1.11 \pm 0.04 \mathrm{nmol} \mathrm{mg}_{\text {prot }}{ }^{-1}$ ) at the same level (500 $\left.\mathrm{mg} \mathrm{L}^{-1}, p<0.05\right)$. Even the low level treatment group $\left(10 \mathrm{mg} \mathrm{L}^{-1}\right.$ ) of VA (content of MDA, $0.98 \pm 0.02 \mathrm{nmol} \mathrm{mg}_{\text {prot }}{ }^{-1}$ ) was more powerful than the Trolox group, although no significant difference was found.

As one of the most important non-enzymatic antioxidant defenses within the cell, GSH provides the first line of defense during oxidative stress; ${ }^{32}$ thus, the levels of GSH and its oxide form GSSG are major oxidative stress markers in cells. As shown in Fig. 6B, the highest GSSG level $\left(1.63 \pm 0.02 \mathrm{nmol} \mathrm{mg}_{\text {prot }}{ }^{-1}\right)$ and lowest GSH level $\left(0.11 \pm 0.01 \mathrm{nmol} \mathrm{mg}_{\text {prot }}{ }^{-1}\right)$, were all obtained from the AAPH group, as expected. Compared to the levels of GSSG and GSH of the AAPH group, the levels of GSSG and GSH of the sample groups incubated with medium (500 $\left.\mathrm{mg} \mathrm{L}^{-1}\right)$ or high levels $\left(1000 \mathrm{mg} \mathrm{L}^{-1}\right)$ were significantly decreased by $58.83-80.83 \%$ and increased by $17-27$ times, respectively $(p<0.05)$. In particular, no significant difference in the GSSG content was obtained between high level treatment groups (in a range from $0.31 \pm 0.02$ to $0.34 \pm 0.02 \mathrm{nmol} \mathrm{mg}_{\text {prot }}{ }^{-1}$ ) and the control group $\left(0.35 \pm 0.02 \mathrm{nmol} \mathrm{\textrm {mg } _ { \text { prot } }}{ }^{-1}\right)$. As for GSH content, the control group (3.02 $\pm 0.01 \mathrm{nmol} \mathrm{mg}$ prot $\left.^{-1}\right)$ showed remarkably higher content than the high level treatment group 

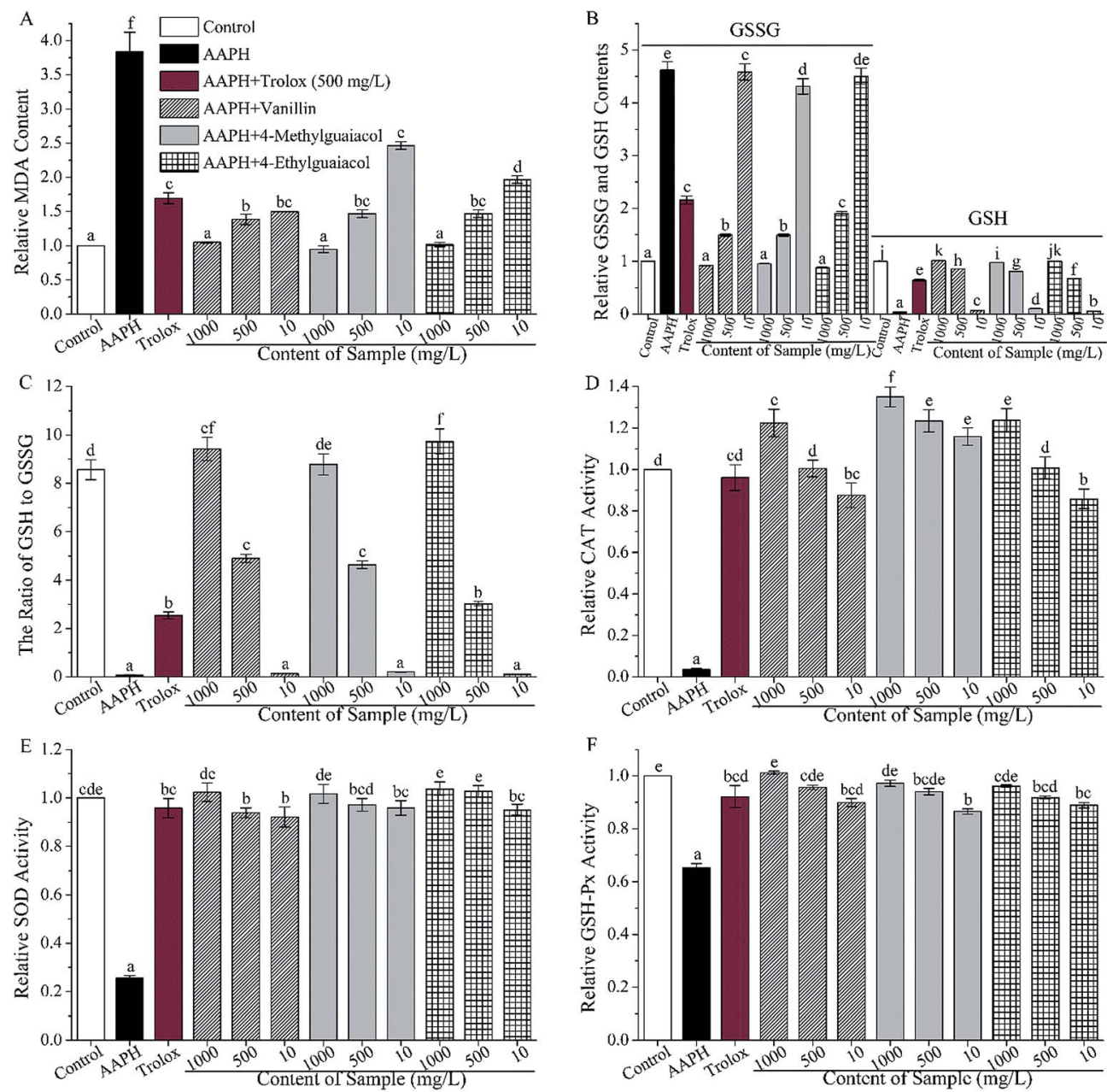

Fig. 6 Effects of vanillin, 4-methylguaiacol, and 4-ethylguaiacol on AAPH-induced changes in MDA (A), GSSG and GSH (B), the GSH/GSSG ratio (C), CAT (D), SOD (E), and GSH-Px levels (F) of HepG2 cells. The results are expressed as relative values versus the control group. Data are shown as the mean \pm SD from three independent experiments. Different letters represent the significant difference at $p<0.05$.

of 4-MG $\left(2.95 \pm 0.01 \mathrm{nmol} \mathrm{mg}_{\text {prot }}^{-1}, p<0.05\right)$ and lower than the high level treatment group of VA $\left(3.07 \pm 0.01 \mathrm{nmol} \mathrm{mg}_{\text {prot }}{ }^{-1}\right.$, $p<0.05)$ and 4-EG $\left(3.04 \pm 0.01 \mathrm{nmol} \mathrm{mg}_{\text {prot }}^{-1}, p>0.05\right)$. Moreover, at the same level $\left(500 \mathrm{mg} \mathrm{L^{-1 }}\right)$, the GSH contents of sample groups (in a range from $(2.03 \pm 0.01$ to $2.58 \pm 0.01 \mathrm{nmol} \mathrm{mg}_{\text {prot }}{ }^{-1}$ ) were all significantly higher than that of the Trolox group $\left(1.93 \pm 0.04 \mathrm{nmol} \mathrm{mg}_{\text {prot }}{ }^{-1}, p<0.05\right)$. Apart from the 4-EG group (GSSG content, $0.67 \pm 0.02 \mathrm{nmol} \mathrm{mg}_{\text {prot }}{ }^{-1}$ ), the GSSG contents of VA $\left(0.53 \pm 0.02 \mathrm{nmol} \mathrm{mg}_{\text {prot }}{ }^{-1}\right)$ and 4-MG groups $\left(0.54 \pm 0.02 \mathrm{nmol} \mathrm{mg}\right.$ prot $\left.^{-1}\right)$ were all significantly lower than that of the Trolox group $\left(0.76 \pm 0.03 \mathrm{nmol} \mathrm{mg}\right.$ prot $^{-1}$, $p<0.05$ ).

In order to better understand the influence of VA, 4-MG, and 4-EG on AAPH-induced changes in GSSG and GSH levels of HepG2 cells, the ratio of GSH to GSSG was also assessed. As shown in Fig. 6C, undoubtedly, the AAPH group exhibited the lowest ratio of GSH to GSSG, and no significant difference was found between low level treatment groups and the AAPH group $(p>0.05)$. The results indicate that these groups had higher oxidative stress, and low level treatment of VA, 4-MG, and 4-EG did not show any protective effects on AAPH-induced changes in the GSH level of HepG2 cells. In contrast to the AAPH group, high level and medium level treatment groups significantly increased the ratio of GSH to GSSG $(p<0.05)$, particularly the high level treatment groups, the ratios of which (in a range from $8.78 \pm 0.44$ to $9.73 \pm 0.52$ ) were all higher than those of the control group $(8.56 \pm 0.41)$. It was observed that the protective effects on AAPH-induced changes in the GSH level of VA, 4-MG, and 4-EG exhibited dose-dependence. Also noted was that the GSH/GSSG ratios of sample groups were all significantly higher than those of the Trolox group $(2.54 \pm 0.14, p<0.05)$ at the same level $\left(500 \mathrm{mg} \mathrm{L}^{-1}\right)$, except for those of the 4-EG group $(3.03 \pm 0.09)$.

Several enzymatic and non-enzymatic mechanisms balance the production of ROS and transform ROS into non-toxic molecules. ${ }^{33}$ Among them, CAT, SOD and GSH-Px are the key participants of antioxidant defense systems in cells. CAT and GSH-Px reduce the production of ROS. SOD converts superoxide into hydrogen peroxide, which is transformed into water and oxygen by CAT and GSH-Px. ${ }^{34}$ Depletion in the activities of these antioxidant enzymes can be due to enhanced oxidative stress; therefore, the activities of CAT, SOD and GSH-Px were 
determined in this study. As shown in Fig. 6D, the weakest CAT activity $\left(0.71 \pm 0.12 \mathrm{U} \mathrm{mg}_{\text {prot }}{ }^{-1}\right)$ was found in the AAPH group; however, compared to this group, even the low level treatment groups could critically enhance the CAT activity (in the range from $16.99 \pm 0.25$ to $22.95 \pm 0.44 \mathrm{U} \mathrm{mg}_{\text {prot }}{ }^{-1}$ ), which was significantly weakened by AAPH-induced ROS $(p<0.05)$. Furthermore, the CAT activity was returned to normal value (control group, $19.83 \pm 1.07 \mathrm{U} \mathrm{mg}_{\text {prot }}{ }^{-1}$ ) by medium level treatment of the sample (in a range from $19.90 \pm 0.44$ to $24.45 \pm$ $0.54 \mathrm{U} \mathrm{mg}_{\text {prot }}{ }^{-1}$ ) and that of high level treatment groups (in a range from $24.24 \pm 0.25$ to $26.72 \pm 0.62 \mathrm{U} \mathrm{mg}_{\text {prot }}{ }^{-1}$ ) significantly exceeded that of the control group $(p<0.05)$. With respect to the different samples, 4-MG was most effective in protecting the CAT activity against ROS induced by AAPH (CAT activity of the high level treatment group $\left(1000 \mathrm{mg} \mathrm{L}^{-1}\right), 26.72 \pm 0.62 \mathrm{U}$ $\left.\mathrm{mg}_{\text {prot }}^{-1}\right)$, followed by 4-EG $\left(24.52 \pm 0.64 \mathrm{U} \mathrm{mg}_{\text {prot }}{ }^{-1}\right)$ and VA $\left(24.24 \pm 0.25 \mathrm{U} \mathrm{mg}_{\text {prot }}{ }^{-1}\right)$. Although a significant difference in the CAT activity was only found between Trolox and 4-MG groups $(p<0.05)$, all sample groups exhibited stronger protective abilities toward CAT activity (in a range from $19.90 \pm 0.44$ to $\left.24.45 \pm 0.54 \mathrm{U} \mathrm{mg}_{\text {prot }}{ }^{-1}\right)$ than the Trolox group $(19.00 \pm 0.21 \mathrm{U}$ $\mathrm{mg}_{\text {prot }}^{-1}$ ) at the same level (500 $\left.\mathrm{mg} \mathrm{L}^{-1}\right)$.

The result of SOD activity is shown in Fig. 6E. Although SOD activity of the AAPH group $\left(5.90 \pm 0.31 \mathrm{U} \mathrm{mg}_{\text {prot }}{ }^{-1}\right)$ decreased remarkably, the low level treatment groups could significantly improve the SOD activity (in a range from $21.28 \pm 0.22$ to $22.15 \pm 0.30 \mathrm{U} \mathrm{mg}_{\text {prot }}{ }^{-1}$ ), almost 4 times that of the AAPH group $(p<0.05)$. As for different samples, 4-EG exhibited the strongest protection ability on SOD activity $\left(24.00 \pm 0.47 \mathrm{U} \mathrm{mg}_{\text {prot }}{ }^{-1}\right)$ in the high level treatment group (1000 $\left.\mathrm{mg} \mathrm{L}^{-1}\right)$, followed by VA $\left(23.65 \pm 0.08 \mathrm{U} \mathrm{mg}_{\text {prot }}{ }^{-1}\right)$ and $4-\mathrm{MG}\left(23.48 \pm 0.55 \mathrm{U} \mathrm{mg}_{\text {prot }}{ }^{-1}\right)$. Furthermore, although the SOD activities of $4-\mathrm{MG}(22.44 \pm 0.31$ $\left.\mathrm{U} \mathrm{mg}_{\text {prot }}{ }^{-1}\right)$ and 4-EG groups $\left(23.77 \pm 0.50 \mathrm{U} \mathrm{mg}_{\text {prot }}{ }^{-1}\right)$ were all higher than that of the Trolox group $\left(22.13 \pm 0.24 \mathrm{U} \mathrm{mg}_{\text {prot }}{ }^{-1}\right)$ at the same level $\left(500 \mathrm{mg} \mathrm{L}^{-1}\right)$, the significant difference in the SOD activity was only found between Trolox and the 4-EG groups $(p<0.05)$. Moreover, the SOD activity of the VA group $\left(21.67 \pm 0.51 \mathrm{U} \mathrm{mg}_{\text {prot }}{ }^{-1}\right)$ was lower than that of the Trolox group $(p>0.05)$.

As shown in Fig. 6F, AAPH-induced ROS had less influence on the activity of GSH-Px compared to those of CAT and SOD. VA, 4-MG, and 4-EG all showed significant protective effects to increase the activity of GSH-Px (in the range from $518.65 \pm 10.08$ to $606.92 \pm 16.14 \mathrm{U} \mathrm{mg}_{\text {prot }}{ }^{-1}$ ), compared to that of the AAPH group (392.35 $\left.\pm 14.90 \mathrm{U} \mathrm{mg}_{\text {prot }}{ }^{-1}, p<0.05\right)$. Although VA registered the highest protective effect and 4-EG obtained the lowest protective effect, no significant difference in the GSH-Px activity was found for HepG2 cells pretreated with different samples. Despite the fact that VA and 4-MG exerted stronger protective effects on the GSH-Px activity than Trolox, and 4-EG showed weaker protective effects than Trolox at the same level (500 $\mathrm{mg} \mathrm{L}^{-1}$ ), no significant difference was found.

\section{Discussion}

Recently, studies have identified that phenolic compounds have been shown to possess certain health-promoting characteristics based on their potential antioxidant effects. ${ }^{34}$ However, the relationship between the phenolic aromatic compounds of Chinese Baijiu and human health still confused people. Vanillin (VA), 4-methylguaiacol (4-MG), and 4-ethylguaiacol (4EG), three phenolic aroma compounds of Chinese Baijiu, were therefore selected to evaluate their antioxidant activities.

For in vitro chemical assays, it is worth mentioning that VA, 4-MG, and 4-EG exhibited different antioxidant activities in different assays. VA, 4-MG, and 4-EG exhibited stronger antioxidant activities than Trolox in ABTS, ORAC, and reducing power assays, particularly in the ABTS assay, but showed weak activity in the DPPH assay and no activity in $\mathrm{Fe}^{2+}$ chelating ability assay. It has been reported that VA showed strong radical scavenging activity in the ABTS assay, but little activity in the DPPH assay, ${ }^{35}$ which is consistent with this study. The extremely high activity of 4-MG and 4-EG shown in the ABTS assay might be due to self-dimerization. Reportedly, although 1 mole of VA has only one oxidizable phenolic hydroxyl group, it can scavenge more than one equivalent of free radicals via adduct formation with the radical, and the oxidative dimer formation contributes to the total radical-scavenging ability of VA. ${ }^{24}$ 4-MG and 4-EG displayed greater radical scavenging activity than VA, which indicated that 4-MG and 4-EG reacted with radicals via self-dimerization in the same manner as VA, and hence 1 mole of 4-MG or 4-EG could scavenge more than one equivalent of free radical. In addition, it has been reported that the efficiency of guaiacol derivatives as radical scavengers is associated with the nature of the group in the para position to phenolic $\mathrm{OH}^{3}$ Those guaiacol derivatives with electro-donating groups (vanillic alcohol and eugenol) were more reactive than guaiacol, and those with electron-withdrawing groups (vanillin and vanillic acid) were less reactive than guaiacol in non-polar environments. ${ }^{3}$ Therefore, the higher radical scavenging activity of 4MG and 4-EG, with respect to VA, could be explained by the presence of the alkyl group $\left(-\mathrm{CH}_{3}\right.$ and $\left.-\mathrm{CH}_{2} \mathrm{CH}_{3}\right)$ in the para position to the phenolic $\mathrm{OH}$ (shown in Fig. 1). The alkyl group was the electron-donating group, while the aldehyde group bonded directly to an aromatic hydrocarbon atom was the electron-withdrawing group. It was also noted that the different antioxidant activities of the samples were shown in different assays. For example, the TE value of VA was higher than the others in the ORAC and reducing power assays, but lower than the others in the DPPH and ABTS assays. Different radical sources, substrates, reaction conditions, and mechanisms seemed to be responsible for the different results of in vitro chemical assays. Regarding the antioxidant activity of guaiacol and vanillin, three reaction mechanisms have been proposed: the hydrogen transfer (HT) from the phenolic $\mathrm{OH},{ }^{36-38}$ the single electron transfer (SET) from the guaiacol and vanillin to the radical, ${ }^{\mathbf{8} 39,40}$ and sequential proton electron transfer (SPET) mechanism. ${ }^{3}$ Moreover, it was found that the environment plays an important role in the peroxyl scavenging activity of guaiacol and vanillin. ${ }^{3}$ Therefore, in order to completely evaluate the antioxidant activities, it was essential to apply different assay systems. To sum up, 4-ethylguaiacol exhibited good antioxidant activity in all vitro chemical assays, except the $\mathrm{Fe}^{2+}$ chelating ability assay, followed by 4-methylguaiacol and vanillin. 
Reports of the direct antioxidant effects of guaiacol and its analogs (i.e., vanillin and ethyl vanillin) in in vitro chemical assays are available; ;,19,22 vanillin, 4-methylguaiacol, and 4-ethylguaiacol also exerted outstanding in vitro antioxidant activities in this experiment, but vitro chemical assays are significantly different from physiological assays. Since hepatocytes are equipped with molecular strategies that maintain balance between the oxidant and antioxidant systems, the HepG2 cell model was taken to test the intracellular antioxidant activities of vanillin, 4-methylguaiacol, and 4-ethylguaiacol. In this study, ROS that was triggered by AAPH was produced abundantly in HepG2 cells, while VA, 4-MG, and 4-EG could relieve the increased oxidative stress quickly (within $30 \mathrm{~min}$ ) and efficiently maintain the ROS amount of sample groups lower than that of the AAPH group within $180 \mathrm{~min}$. The ROS of the low level treatment groups increased again due to the exhaustion of the sample. However, the high level of sample treatment could maintain the ROS at normal levels. These findings indicate that VA, 4-MG, and 4-EG exerted their protective effects in a dose-dependent manner, consistent with the collective evidence that phenolic compounds in foods and beverages can suppress the oxidative stress induced by enhanced ROS. ${ }^{13}$ VA, 4-MG, and 4-EG were probably more effective than Trolox due to the fact that the ROS amounts of sample groups were significantly lower than that of the Trolox group after $60 \min (p<0.05)$. In addition, interestingly, the ORAC assay and HepG2 cell model used the same radical source, AAPH-derived peroxyl radicals, and the results of these assays were therefore expected to be correlated with each other to some extent. However, the protective effect of VA was the highest in the ORAC assay, and lowest in the HepG2 cell model. Similar results were found in the ORAC assay and oxidative hemolysis inhibition assay (OxHLIA), ${ }^{22}$ which suggest that the environment had a strong influence on peroxyl scavenging activity of VA.

Previous studies have demonstrated that enhanced ROS would decrease the capacity of the intracellular antioxidant system, affect major cellular components (i.e., lipids, DNA and proteins), and accelerate cell apoptosis in vivo. ${ }^{41,42}$ The intracellular oxidative stress is directly linked to the important antioxidant enzymes (i.e., CAT, SOD, and GSH-Px) ${ }^{43}$ which generally indicate the capacity of the antioxidant defense system and is usually reflected by the levels of MDA, GSH and GSSG (oxidative stress markers in cells). ${ }^{44}$ In this study, increased oxidative stress, generated by AAPH, generally caused a rise in MDA and GSSG content and a decrease in CAT, SOD and GSH-Px activities as well as GSH content. However, VA, 4MG, and 4-EG could markedly improve AAPH-induced alterations in oxidative stress biomarkers and antioxidant enzymes, even more than Trolox. According to the results of previous studies ${ }^{42,45}$ the increased generation of MDA and GSSG and the decreased content of GSH were suppressed by phenolic compounds, which were confirmed in this experiment. The contents of MDA and GSSG were significantly higher in the AAPH group when compared with the control group $(p<0.05)$; it was also noted that with the ROS increasing, GSH was exhausted and converted into its oxidized forms (GSSG). However, it could be observed that high levels of sample treatment not only completely suppressed the formation of MDA, but also significantly enhanced the ratio of GSH to GSSG, compared to those of the control group ( $p<0.05$ ), which suggested that VA, 4-MG, and 4-EG could protect HepG2 cells against AAPH-induced ROS by themselves and induce the formation of GSH. Likewise, phenolic compound (from red raspberry) treatment of HepG2 cells enhanced the total cellular GSH levels and dramatically increased the GSH/GSSG ratio. ${ }^{46}$ In addition, it has been reported that phenolic compounds not only act as antioxidants themselves, but also modulate or induce other intracellular antioxidative activity (i.e., CAT, SOD, and GSH-Px). ${ }^{15,47}$ In this study, it was observed that AAPH significantly suppressed the activities of CAT, SOD, and GSH-Px $(p<0.05)$; however, the pretreatment of AAPH-damaged HepG2 cells with VA, 4-MG, and 4-EG effectively increased the activities of SOD, CAT, and GSH-Px $(p<0.05)$. Similar findings were obtained from previous reports; phenolic compounds were found to possess antioxidant capacity, and protected the cells from oxidative stress damage through improving the activities of important intracellular antioxidant enzymes (i.e., CAT, SOD, and GSH-Px). ${ }^{45,48} \mathrm{It}$ is also worth noting that even the low level treatment group (10 $\mathrm{mg} \mathrm{L}^{-1}$ ) of 4-MG had significantly higher CAT activity than that of the control group $(p<0.05)$. Furthermore, the activity of CAT was more sensitive to the ROS induced by AAPH, compared to those of SOD and GSH-Px, which is due to the fact that the GSH-Px activity of the AAPH group only decreased by $34.59 \%$; however, the CAT activity of the AAPH group was near zero and decreased by $96.43 \%$. Taken together, although the antioxidant activities of vanillin, 4-methylguaiacol, and 4-ethylguaiacol differed in varied in vitro antioxidant systems, they exhibited similar antioxidant activities in HepG2 cells treated with AAPH due to their similar chemical structures.

\section{Conclusions}

The antioxidant activities of vanillin, 4-methylguaiacol, and 4ethylguaiacol, three aromatic compounds found in Chinese Baijiu, were evaluated by in vitro chemical assays and the HepG2 cell model. Vanillin, 4-methylguaiacol, and 4-ethylguaiacol exhibited stronger antioxidant activities than Trolox in ABTS, ORAC, and reducing power assays, particularly in the ABTS assay. In the AAPH-induced HepG2 cell model, vanillin, 4methylguaiacol, and 4-ethylguaiacol exerted similar antioxidant activities in a dose-dependent manner. They relieved the increased oxidative stress quickly and efficiently by inhibiting the formation of MDA, enhancing the activities of CAT, SOD and GSH-Px and inducing the formation of GSH. These results could lay the foundation for better illustrating the health protective properties of Chinese Baijiu.

\section{Conflicts of interest}

No potential conflict of interest was reported by the authors. 


\section{Acknowledgements}

This work was supported by the National Key R\&D Program of China [2016YFD0400501] and the National Natural Science Foundation of China [31301466, 31601556].

\section{Notes and references}

1 K. Ganesan and B. Xu, A critical review on polyphenols and health benefits of black soybeans, Nutrients, 2017, 9, 455-471.

2 M. Xu, R. Liang, Y. Li and J. Wang, Anti-fatigue effects of dietary nucleotides in mice, Food Nutr. Res., 2017, 61, 1334485.

3 A. Galano, J. R. Leoón-Carmona and J. R. Alvarez-Idaboy, Influence of the environment on the protective effects of guaiacol derivatives against oxidative stress: mechanisms, kinetics, and relative antioxidant activity, J. Phys. Chem. B, 2012, 116, 7129-7137.

4 J. K. Willcox, S. L. Ash and G. L. Catignani, Antioxidants and prevention of chronic disease, Crit. Rev. Food Sci. Nutr., 2004, 44, 275-295.

5 M. Valko, H. Morris and M. T. D. Cronin, Metals, toxicity and oxidative stress, Curr. Med. Chem., 2005, 12, 1161-1208.

6 P. Pasupathi, Y. Y. Rao, J. Farook, G. Saravanan and G. Bakthavathsalam, Effect of cigarette smoking on lipids and oxidative stress biomarkers in patients with acute myocardial infarction, Res. J. Med. Med. Sci., 2009, 4, 15l-159.

7 J. R. Reiter, C. L. Manchester and D. X. Tan, Neurotoxins: free radical mechanisms and melatonin protection, Curr. Neuropharmacol., 2010, 8, 194-210.

8 I. Lassoued, L. Mora, R. Nasri, M. Jridi, F. Toldrá, M. C. Aristoy, A. Barkia and M. Nasri, Characterization and comparative assessment of antioxidant and ACE inhibitory activities of thornback ray gelatin hydrolysates, J. Funct. Foods, 2015, 13, 225-238.

9 G. Buonocore, S. Perrone and M. L. Tataranno, Oxygen toxicity: chemistry and biology of reactive oxygen species, Semin. Fetal Neonatal Med., 2010, 15, 186-190.

10 A. Ljevar, N. Ćurko, M. Tomašević, K. Radošević, V. G. Srček and K. K. Ganić, Phenolic composition, antioxidant capacity and in vitro cytotoxicity assessment of fruit wines, Food Technol. Biotechnol., 2016, 54, 145-155.

11 Y. Chen, G. Chen, X. Fu and R. Liu, Phytochemical profiles and antioxidant activity of different varieties of Adinandra tea (Adinandra Jack), J. Agric. Food Chem., 2014, 63, 169-176.

12 H. J. Jung, Y. S. Song, K. Kim, C. Lim and E. Park, Assessment of the anti-angiogenic, anti-inflammatory and antinociceptive properties of ethyl vanillin, Arch. Pharmacal Res., 2010, 33, 309-316.

13 M. S. Lingua, M. P. Fabani, D. A. Wunderlin and M. V. Baroni, In vivo antioxidant activity of grape, pomace and wine from three red varieties grown in Argentina: Its relationship to phenolic profile, J. Funct. Foods, 2016, 20, 332-345.

14 R. H. Soliman, O. A. Ismail, M. S. Badr and S. M. Nasr, Resveratrol ameliorates oxidative stress and organ dysfunction in Schistosoma mansoni infected mice, Exp. Parasitol., 2017, 174, 52-58.

15 M. Tanno, A. Kuno, T. Yano, T. Miura, S. Hisahara, S. Ishikawa, K. Shimamoto and Y. Horio, Induction of manganese superoxide dismutase by nuclear translocation and activation of SIRT1 promotes cell survival in chronic heart failure, J. Biol. Chem., 2010, 285, 8375-8382.

16 G. Cunha, V. Silva, K. Bessa, M. Bitencourt, U. Macêdo, F. Freire-Neto, R. Martins, C. Assis, T. Lemos, M. Almeida and A. Freire, Levels of oxidative stress markers: correlation with hepatic function and worm burden patients with schistosomiasis, Acta Parasitol., 2012, 57, 160-166.

17 B. Sun, J. Wu, M. Huang, J. Sun and F. Zheng, Recent advances of flavor chemistry in Chinese liquor spirits (Baijiu), J. Chin. Inst. Food Sci. Technol., 2015, 15, 1-8.

18 X. W. Zheng and B. Z. Han, Baijiu, Chinese liquor: history, classification and manufacture, Journal of Ethnic Foods, 2016, 3, 19-25.

19 K. G. Lee, S. E. Lee, G. R. Takeoka, J. H. Kim and B. S. Park, Antioxidant activity and characterization of volatile constituents of beechwood creosote, J. Sci. Food Agric., 2005, 85, 1580-1586.

20 H. J. Yang, J. H. Lee, K. Y. Lee and K. B. Song, Antimicrobial effect of an Undaria pinnatifida composite film containing vanillin against Escherichia coli and its application in the packaging of smoked chicken breast, Int. J. Food Sci. Technol., 2017, 52, 398-403.

21 R. M. Cava-Roda, A. Taboada-Rodríguez, M. T. ValverdeFranco and F. Marín-Iniesta, Antimicrobial activity of vanillin and mixtures with cinnamon and clove essential oils in controlling Listeria monocytogenes and Escherichia coli O157: H7 in milk, Food Bioprocess Technol., 2012, 5, 2120-2131.

22 A. Tai, T. Sawano and F. Yazama, Antioxidant properties of ethyl vanillin in vitro and in vivo, Biosci., Biotechnol., Biochem., 2011, 75, 2346-2350.

23 A. A. Abdulrahman, K. Faisal, A. A. A. Meshref and M. Arshaduddin, Low-dose acute vanillin is beneficial against harmaline-induced tremors in rats, Neurol. Res., 2017, 39, 264-270.

24 J. R. O. Neto, T. S. de Oliveira, P. C. Ghedini, B. G. Vaz and E. de Souza Gil, Antioxidant and vasodilatory activity of commercial beers, J. Funct. Foods, 2017, 34, 130-138.

25 L. Zheng, Y. Zhao, H. Dong, G. Su and M. Zhao, Structureactivity relationship of antioxidant dipeptides: dominant role of Tyr, Trp, Cys and Met residues, J. Funct. Foods, 2016, 21, 485-496.

26 J. Takebayashi, Y. Yagi, R. Ishii, S. Abe, K. Yamada and A. Tai, Antioxidant properties of 2-O- $\beta$-D-glucopyranosyl-L-ascorbic acid, Biosci., Biotechnol., Biochem., 2008, 72, 1558-1563.

27 X. L. Jin, K. Wang, Q. Q. Li, W. L. Tian, X. F. Xue, L. M. Wu and F. L. Hu, Antioxidant and anti-inflammatory effects of Chinese propolis during palmitic acid-induced lipotoxicity in cultured hepatocytes, J. Funct. Foods, 2017, 34, 216-223.

28 R. L. Prior, X. Wu and K. Schaich, Standardized methods for the determination of antioxidant capacity and phenolics in 
foods and dietary supplements, J. Agric. Food Chem., 2005, 53, 4290-4302.

29 L. Zheng, H. Dong, G. Su, Q. Zhao and M. Zhao, Radical scavenging activities of Tyr-, Trp-, Cys- and Met-Gly and their protective effects against AAPH-induced oxidative damage in human erythrocytes, Food Chem., 2016, 197, 807-813.

30 M. Y. B. Çimen, Free radical metabolism in human erythrocytes, Clin. Chim. Acta, 2008, 390, 1-11.

$31 \mathrm{P}$. Ambigaipalan and F. Shahidi, Bioactive peptides from shrimp shell processing discards: antioxidant and biological activities, J. Funct. Foods, 2017, 34, 7-17.

32 C. G. Zou, N. S. Agar and G. L. Jones, Oxidative insult to human red blood cells induced by free radical initiator AAPH and its inhibition by a commercial antioxidant mixture, Life Sci., 2001, 69, 75-86.

33 F. J. Giordano, Oxygen, oxidative stress, hypoxia, and heart failure, J. Clin. Invest., 2005, 115, 500-508.

34 R. Mattera, M. Benvenuto, M. G. Giganti, I. Tresoldi, F. R. Pluchinotta, S. Bergante, G. Tettamanti, L. Masuelli, V. Manzari, A. Modesti and R. Bei, Effects of polyphenols on oxidative stress-mediated injury in cardiomyocytes, Nutrients, 2017, 9, 523-565.

35 A. Tai, T. Sawano, F. Yazama and H. Ito, Evaluation of antioxidant activity of vanillin by using multiple antioxidant assays, Biochim. Biophys. Acta, 2011, 1810, 170177.

36 E. Anouar, C. A. Calliste, P. Kosinova, F. Di Meo, J. L. Duroux, Y. Champavier, K. Marakchi and P. Trouillas, Free radical scavenging properties of guaiacol oligomers: A combined experimental and quantum study of the guaiacyl-moiety role, J. Phys. Chem. A, 2009, 113, 13881-13891.

37 S. Fujisawa, T. Atsumi, Y. Kadoma and H. Sakagami, Antioxidant and prooxidant action of eugenol-related compounds and their cytotoxicity, Toxicology, 2002, 177, 39-54.

38 S. A. Ordoudi, M. Z. Tsimidou, A. P. Vafiadis and E. G. Bakalbassis, Structure-DPPH ${ }^{*}$ scavenging activity relationships: parallel study of catechol and guaiacol acid derivatives, J. Agric. Food Chem., 2006, 54, 5763-5768.
39 S. N. Guha and K. I. Priyadarsini, Kinetic and redox characteristics of phenoxyl radicals of eugenol and isoeugenol: a pulse radiolysis study, Int. J. Chem. Kinet., 2000, 32, 17-23.

40 H. S. Mahal, L. P. Badheka and T. Mukherjee, Radical scavenging properties of a flavouring agent-Vanillin, Res. Chem. Intermed., 2001, 27, 595-604.

$41 \mathrm{H}$. Cichoż-Lach and A. Michalak, Oxidative stress as a crucial factor in liver diseases, World J. Gastroenterol., 2014, 20, 8082-8091.

42 J. Wang, B. Sun, Y. Cao and C. Wang, Wheat bran feruloyl oligosaccharides enhance the antioxidant activity of rat plasma, Food Chem., 2010, 123, 472-476.

43 R. J. Elias, S. S. Kellerby and E. A. Decker, Antioxidant activity of proteins and peptides, Crit. Rev. Food Sci. Nutr., 2008, 48, 430-441.

44 S. Bagis, L. Tamer, G. Sahin, R. Bilgin, H. Guler, B. Ercan and C. Erdogan, Free radicals and antioxidants in primary fibromyalgia: an oxidative stress disorder, Rheumatol. Int., 2005, 25, 188-190.

45 M. Zhao, Q. Yang, L. Lin, B. Sun and Y. Wang, Intracellular antioxidant activities of selected cereal phenolic extracts and mechanisms underlying the protective effects of adlay phenolic extracts on $\mathrm{H}_{2} \mathrm{O}_{2}$-induced oxidative stress in human erythrocytes, J. Funct. Foods, 2017, 31, 160-171.

46 S. Anwar, A. Speciale, D. Fratantonio, M. Cristani, A. Saija, F. Virgili and F. Cimino, Cyanidin-3-O-glucoside modulates intracellular redox status and prevents HIF-1 stabilization in endothelial cells in vitro exposed to chronic hypoxia, Toxicol. Lett., 2014, 226, 206-213.

47 B. O. Cho, H. W. Ryu, C. W. Lee, C. H. Jin, W. D. Seo, J. Ryu, D. S. Kim, S. Y. Kang, H. S. Yook and I. Y. Jeong, Protective effects of new blackberry cultivar MNU-32 extracts against $\mathrm{H}_{2} \mathrm{O}_{2}$-induced oxidative stress in HepG2 cells, Food Sci. Biotechnol., 2015, 24, 643-650.

48 H. Teng, T. Fang, Q. Lin, H. Song, B. Liu and L. Chen, Red raspberry and its anthocyanins: Bioactivity beyond antioxidant capacity, Trends Food Sci. Technol., 2017, 66, 153-165. 